\title{
The Effect of Taijiquan Exercise on Low Back Muscle Strength in Patients with Primary Osteoporosis
}

\author{
Qiang Liu, Longling Xiao \\ School of Physical Education, Wenshan University, Wenshan Yunnan 663099, China
}

1124286@qq.com

Keywords: Tai Chi Chuan exercise, primary osteoporosis, low back muscle strength, improvement.

\begin{abstract}
To study the effect of Taijiquan Exercise on low back muscle strength in patients with primary osteoporosis. Methods: select our hospital in 20132015 May 5 months admitted to hospital for treatment and rehabilitation of 80 patients with primary osteoporosis patients, the consent of the patient, assigned to experimental group (40 cases) and control group (40 cases), the control group were taken conventional gait training rehabilitation, once a day, every Saturday, in six months. Patients in the experimental group take Taijiquan exercise method; take the Chen Style Taijiquan exercise as the main movement foundation, training time and the control group of the same. After training, the clinical manifestations of the two groups were compared, including the main testing standards in the lumbar flexion and extension before and after training, as well as lumbar muscle strength test. Results: Taijiquan stem the prognosis of the patients in the clinical situation were significantly higher than those of the control group patients recovery, waist stretch and curvature, are significantly better than the control group, and treatment of patients in the two groups of data by statistical analysis found that, $\mathrm{P}<0.05)$. Conclusion: Tai Chi exercise method for patients with primary osteoporosis was therapy resulted in clinical improvement, with a significant and better clinical effect can be applied and extended in the clinical treatment of primary osteoporosis.
\end{abstract}

\section{Introduction}

Primary osteoporosis is a typical clinical symptoms of osteoporosis, high incidence, morbidity higher, which give the society and the family has brought great economic losses. In the treatment of this type disease clinically, we usually use the method of choice to drug combination therapy and exercise to improve the condition [1-2]. Many studies in the country mentioned, may provide a better environment for the body of the patient through effective exercise methods in the use of exercise methods. While the application of this case, the blood flow to the cortical bone and the improvement of bone mineral density also play a certain effect [3-4]. Primary osteoporosis clinical symptoms in excitation, the biggest threat comes secondary fractures, over ninety percent of these diseases fractures during the study. We briefly discussed this type of disease in our hospital. Comparison Taijiquan with conventional clinical vigorous training for our elderly patients with primary osteoporosis improve the situation, reported as follows in the process of the exercise assisting in governance.

\section{Experimental Procedure}

Object of study. The experiment data sample clinical study admitted to our hospital rehabilitation 80 patients with primary osteoporosis from May 2013 to May 2015, which patients are in line with the Chinese people into the bone delivery symptom diagnostic criteria, and all patients were voluntarily participated in this clinical experimental study. Before attending the hospital treatment, all patients had no regular exercise habits. All patients were excluded secondary osteoporosis cases, and do not include patients with a history of secondary fractures, in them no containing hormone replacement drug treatment history. After patient consent, they will be divided into a control group of 40 patients, 16 males and 24 females, mean age $62.8 \pm 10.8$ years old, taking vigorous training routine rehabilitation once a day, six times a week for six months in order to prevail; experimental group of 
40 patients, 18 males and 22 females, mean age $63.5 \pm 9.5$ years old, taking Taijiquan exercise method. Taijiquan is taken as the main basis of sports training time with the control group of the same. The basic situation of all patients by comparative analysis, there are no significant difference comparablly, $\mathrm{P}>0.05$, Specific data is as shown in Table 1 below.

Table 1 General information differences between the two groups

\begin{tabular}{ccccc}
\hline Packet Group & \multirow{2}{*}{ Number of cases } & age & $\begin{array}{c}\text { body } \\
\text { weight }\end{array}$ & height \\
\hline & & $63.5 \pm 9.5$ & $58.6 \pm 16.7$ & $159.6 \pm$ \\
test group & 40 & $62.8 \pm$ & $55.6 \pm 14.8$ & $156.5 \pm$ \\
Group & 40 & 10.8 & $>0.05$ & 11.5 \\
P & & $>0.05$ & & $>0.05$ \\
\hline
\end{tabular}

Experimental methods. Control group: patients were treated with $\alpha$-D3 capsules twice daily, once $0.25 \mu \mathrm{g}$, calcium glaciate three times a day, the amount depending on the symptoms the patient to choose. Auxiliary training methods to health as the main method static brisk step, once a day, six times a week for six months as a benchmark exercise. More exercise by professional coaches for guidance.

Experimental groups: standard treatment with the control group of the same, auxiliary exercise places Chen Taijiquan method as the main method of exercise once a day, six times a week for six months as a benchmark exercise, exercise guidance by a professional instructor.

Treatment evaluation standards. After the two groups before the study began its Training and ending in June, respectively, with the United States 3-yield multi-joint System Bide is kinetic testing and rehabilitation systems and supporting the lumbar flexion and extension attachments, use of lumbar flexion Concentric Contraction test program (i.e.,: $60^{\circ} / \mathrm{s} \times 5$ and $90^{\circ} / \mathrm{s} \times 10$ ) were the lumbar flexion and extension muscle strength test.

Test Methods: Subjects were sitting, feet placed on adjustable feet on the board, the dynamometer axis aligned with the subject's fifth lumbar spinouts process, the routine use of straps fixed to the patient on the lumbar flexion and extension annex. So that they underwent three warm-up natures of the Concentric Contraction attempts, began formal testing.

Observation Index: Main movable joint activities of the (active range of motion, AROM), peak torque (peak torque, PT), peak torque weight ratio (peak torque to body weight, PT / BW), to reach the peak torque time (time Topeka torque, TPT), the total work load (total work, TW), lumbar flexion / extension muscle strength ratio value (flexion to extension, $\mathrm{F} / \mathrm{E}$ ).

Statistical methods. The comparative clinical data with statistical software SPSS16.8 statistical analysis as the main program, the chi-square test or $\mathrm{X}^{2}$ places $t$ be detected when $\mathrm{P}<0.05$, the clinical data showed that this result was statistically significant.

\section{Results and discussion}

Results and analysis of both groups' $60 \%$ s angular velocity and kinetic testing. According to Table 2: lower lumbar flexion, in two groups of patients before and after the intervention were significantly improved, which is more obvious improvement in the experimental group, AROM, PT and PT / BW large increase rate than the control group; extended state at the waist, two no significant difference in patients before the experiment, after the test in the experimental group than in the control group was significantly different. The clinical trial statistical analysis of the clinical symptoms of the two groups of patients before and after intervention were significantly different, $\mathrm{P}$ $<0.05$, and the two groups have significant differences in clinical symptoms after treatment situation, namely, $\mathrm{P}<0.05$, by analysis, having Statistical significance. 
Table $260^{\circ}$ s angular velocity and constant velocity test results in two groups of patients

\begin{tabular}{cccccc}
\hline \multirow{2}{*}{ Packet Group } & \multicolumn{3}{c}{ Lumbar flexion } & \multicolumn{2}{c}{ Waist stretch } \\
\cline { 2 - 6 } & AROM & PT & PT/BW & PT & PT/BW \\
\hline & $49.1 \pm 9.5$ & $44.6 \pm$ & $77.5 \pm$ & & \\
Experimental group before & $55.2 \pm$ & 25.9 & 20.3 & $59.2 \pm 20.1$ & $89.5 \pm 41.6$ \\
intervention & 11.5 & $58.6 \pm$ & $82.5 \pm$ & $110.2 \pm$ & $125.6 \pm$ \\
Intervention & $45.3 \pm$ & 20.3 & 19.1 & 10.5 & 45.2 \\
The control group before intervention & 10.3 & $57.2 \pm$ & $75.2 \pm$ & $58.6 \pm 21.2$ & $90.5 \pm 46.5$ \\
Intervention & $50.2 \pm$ & 19.5 & 15.2 & $81.6 \pm 20.4$ & $102.5 \pm$ \\
& 11.5 & $60.2 \pm$ & $79.5 \pm$ & & 30.6 \\
\hline
\end{tabular}

NOTE: clinical symptoms before and after comparison of two groups of patients, $\mathrm{P}<0.05$, after the results of the experimental treatment group compared with the control group, $\mathrm{P}<0.05$.

Angular velocity of $90^{\circ}$ in two groups of patients. According to Table 3, the two groups were $90^{\circ}$ angular velocity clinical detailed comparison of the data are as follows: lower lumbar flexion, two sets of data were significant differences before and after the experiment, two groups of TW magnitude of improvement is not very obvious, the improvement in the experimental group than in the control TPT there were significantly difference; at the waist stretched state, there are significant differences between the experimental and control groups before and after the experiment, in which PT has a very significant difference between the experimental group improved more significantly, PT / BW also significantly change and improve the experimental group situation above in the control group; F / E to change significantly in the experimental group than the control group.

Table 390 comparison of 3 degree /s angular velocity and constant velocity test results between the two groups before and after treatment

\begin{tabular}{cccccc}
\hline Packet Group & \multicolumn{2}{c}{ Lumbar flexion } & \multicolumn{2}{c}{ Waist stretch } & \multirow{2}{*}{ F/E } \\
& TW & TPT & PT & PT/BW & \\
\hline Experimental group before & $49.1 \pm 9.5$ & $44.6 \pm$ & & & $80.2 \pm$ \\
intervention & $55.2 \pm$ & 25.9 & $59.6 \pm 20.1$ & $89.5 \pm 41.6$ & 20.5 \\
Intervention & 11.5 & 20.3 & $110.2 \pm$ & $125.6 \pm$ & $62.6 \pm$ \\
The control group before intervention & $45.3 \pm$ & $57.2 \pm$ & 10.5 & 45.2 & 30.2 \\
Intervention & 10.3 & 19.5 & $58.6 \pm 21.2$ & $90.5 \pm 46.5$ & $79.8 \pm$ \\
& $10.2 \pm$ & $60.2 \pm$ & $81.6 \pm 20.4$ & $102.5 \pm$ & 19.9 \\
& 11.5 & 11.5 & & 30.6 & $78.6 \pm$ \\
& & & & 22.6 \\
\hline
\end{tabular}

Note: The comparison between the results of tests, before and after the experiment $\mathrm{P}<0.05$, the experimental group and control group data difference, $\mathrm{P}<0.05$.

The study, the clinical application of Taijiquan adjuvant therapy clinical rehabilitation treatment in patients with primary osteoporosis, the effect is significant, with better clinical significance.

\section{Discussion}

In the clinical treatment of primary osteoporosis symptoms of relying solely on clinical treatment and rehabilitation of drug, the effect is poor, with little success [5-6]. With our accelerated aging, the incidence of these diseases also provides more whole crowd. For our internal reports, the incidence of such disorders is rising year by year. Compared research results at home and abroad, this type of disease symptoms occurs after Hour, joints, waist and other parts of the muscle groups will appear on the functional activity decreased, its peak torque and weight ratio, etc., will be below normal levels. There are also foreign scholars believe that the decline in physical strength, leading to a vicious cycle 
of exacerbations fundamental emergence of this condition will result in the patient's body to a vicious cycle. In the clinical treatment, rehabilitation training by the surrounding muscles to be able to better ensure their clinical condition reduced to ensure ease the symptoms. In clinical treatment, in order to obtain better results, often we need to strengthen the clinical assisted through training form.

In clinical studies in recent years, the movement has become a major clinical rehabilitation adjuvant therapy, of which vigorous training more traditional Taijiquan and belongs to a more innovative training methods. In previous studies, the report shows that the effect is significant, especially in the use of research-based rehabilitation for patients with osteoporosis [7]. The advantage is that this method can effectively improve the clinical symptoms, do physical fitness, while alleviating clinical symptoms performance situation.

In this clinical study, two groups of patients before the intervention showed no significant clinical difference, and after six months of different exercise, basic clinical groups of patients were generated differences, which compared with before treatment, symptoms effective relief, while the effect of the patient's symptoms remission after comparison, tai chi adjuvant therapy, can better promote the rehabilitation of patients with clinical symptoms. The activities of the effect of Taijiquan, PT and BW treatment were superior to conventional walking effect of the control group. Analysis of the reasons is that Taijiquan own activities on the back have a very good role in promoting their training that the whole body movement of the body, through actions such as spiral wound, to achieve multiple joints throughout the whole body activity, to achieve the whole body a plurality of dirty organ and joint training. Through this clinical trial also confirmed that a combination of training methods Taijiquan, waist stretching exercises for muscle action, compared to the traditional method of walking training, the effect is more pronounced degree of improvement is more pronounced. Clinical treatment of people walking through the training can be achieved by Taiji can get better results. Be analyzed from the biomechanical stability of the lumbar spine, the therapeutic effect of the application of Taijiquan, can effectively maintain the stability of the structure of the lumbar spine, combined with more effective qualitative sports activities, promotion activities on the promotion of low back muscle strength. In the form of sports point of view, this is a better use of the whole body of activities to enhance one's physical activity gymnastics effective for sports training, as well as the role of physical rehabilitation, which have a significant effect. From the point view of social development, also be able to improve the basic quality of life of patients. It belongs to a more scientific exercise.

In summary, the application of Taijiquan method for osteoporosis treatment in patients with clinical improvement, the effect is significant with good clinical results can be popularized in clinical treatment of osteoporosis, it is worth to promote clinical application of rehabilitation therapy.

\section{References}

[1] Zhu Yan, Chen Shu Yan, Wu Yunpeng, etc. Taijiquan affect back muscle strength female osteoporosis patients. Chinese Journal of loose bone, 2014, (1): 45-48.

[2] Gong Min, Zhang Suzhen, Wang Bin Effects of long-term shadowboxing exercise on bone mineral density in the elderly. Chinese Journal of Clinical Rehabilitation, 2003, 7(15): 2238-2239.

[3] Liwen Qin, Huang Xiufeng, Bai Man, etc. Effect of Taijiquan on the quality of life in perimenopausal women with osteoporosis patients. Qilu Nursing, 2010, 16(11): 16-17.

[4] Qiu Ping, Li Yumin, high-Jin, etc. on the elderly bone metabolic markers in motion. Chinese Journal of Rehabilitation Medicine, 2005, 20(5): 340-342.

[5] Chen Yibing, Xiongzheng Ying, Zhou Yong,etc. Research on osteoporosis and exercise prescription for exercise therapeutic effects. Capital Institute of Physical Education, 2002, 14(4): $38-40$. 
[6] Lu Ying, Li Jie, Xiaobin Deng clinical study abroad Taijiquan Situation and Reflections. Chinese Medicine, 2013, 33(12): 1717-1721.

[7] Song Hua. Tai Chi exercise on bone mineral density in patients with osteoporosis and bone metabolism in primary. Journal of Physical Education, 2008, 15(11): 106-108. 